\title{
Pregravid body mass index is associated with early introduction of complementary foods
}

\author{
Ushma J. Mehta, $\mathrm{PhD}^{1}$, Anna Maria Siega-Riz, PhD, RD ${ }^{1,2,5}$, Amy H. Herring, $\mathrm{ScD}^{3,5}$, Linda \\ S. Adair, $\mathbf{P h D}^{1,5}$, and Margaret E. Bentley, $\mathbf{P h D}^{1,4,5}$ \\ ${ }^{1}$ Department of Nutrition, Gillings School of Global Public Health, University of North Carolina, \\ Chapel Hill, NC \\ 2Department of Epidemiology, Gillings School of Global Public Health, University of North \\ Carolina, Chapel Hill, NC \\ ${ }^{3}$ Department of Biostatistics, Gillings School of Global Public Health, University of North Carolina, \\ Chapel Hill, NC \\ ${ }^{4}$ Institute for Global Health and Infectious Diseases, Gillings School of Global Public Health, \\ University of North Carolina, Chapel Hill, NC \\ ${ }^{5}$ Fellow, Carolina Population Center, University of North Carolina, Chapel Hill, NC
}

\begin{abstract}
OBJECTIVE-To determine whether women who entered pregnancy overweight or obese were less likely to follow American Academy of Pediatrics (AAP) guidelines for introducing complementary foods to infants after four months of age. In addition, to explore whether psychological factors accounted for any of the effect of pregravid body mass index (BMI) on age of complementary food introduction.
\end{abstract}

DESIGN-A prospective cohort study from 2001 to 2005 which recruited pregnant women between 15 to 20 gestational weeks with follow-up through 12 months postpartum from University of North Carolina hospitals $(n=550)$.

STATISTICAL METHODS-Multinomial logit models estimated relative risk ratios (RRR). The outcome was age of complementary food introduction, categorized as less than four months of age, four to six, and six months or later (referent). Maternal BMI was categorized as underweight $\left(<18.5 \mathrm{~kg} / \mathrm{m}^{2}\right)$, normal-weight $\left(18.5 \mathrm{~kg} / \mathrm{m}^{2}\right.$ to $\left.24.9 \mathrm{~kg} / \mathrm{m}^{2}\right)$, and overweight/obese $\left(\geq 25.0 \mathrm{~kg} / \mathrm{m}^{2}\right)$. A series of regression analyses tested mediation by psychological factors measured during pregnancy (depressive symptoms, stress and anxiety).

\footnotetext{
(C) 2012 Academy of Nutrition and Dietetics. Published by Elsevier Inc. All rights reserved.

Corresponding Author (no reprints will be available): Dr. Ushma Mehta, Gillings School of Global Public Health, University of North Carolina at Chapel Hill, 2200 McGavran-Greenberg Hall, CB \#7461, Chapel Hill, NC 27599-7461, Phone: 727.776.6310, Fax: 919.966.9159, umehta@email.unc.edu.

Co-Authors:

Dr. Anna Maria Siega-Riz, 2105-A McGavran Greenberg, 135 Dauer Drive, Campus Box 7435, Chapel Hill 27599-7435

Dr. Amy Herring, 3104-D Mcgavran-Greenberg Hall, 135 Dauer Drive, Campus Box 7420, Chapel Hill 27599

Dr. Linda Adair, Carolina Population Center, University of North Carolina at Chapel Hill, CB\# 8120, 408 University Square-East, 123 West Franklin Street, Chapel Hill, NC 27516-2524

Dr. Margaret Bentley, 124 Rosenau Hall, 135 Dauer Drive, Campus Box 7400, Chapel Hill, NC 27599

Publisher's Disclaimer: This is a PDF file of an unedited manuscript that has been accepted for publication. As a service to our customers we are providing this early version of the manuscript. The manuscript will undergo copyediting, typesetting, and review of the resulting proof before it is published in its final citable form. Please note that during the production process errors may be discovered which could affect the content, and all legal disclaimers that apply to the journal pertain.
} 
RESULTS-More than a third of the study population (35.7\% of 550) entered pregnancy overweight/obese. The majority of participants (75.3\%) introduced foods to their infants between four to six months of age. Compared with normal-weight women, those who were overweight/ obese before pregnancy were more likely $[R R R=2.22(1.23,4.01)]$ to introduce complementary foods before the infant was four months old, adjusting for race, education, and poverty status. Depressive symptoms, stress and anxiety did not account for any of the effect of pregravid overweight/obesity on early food introduction.

CONCLUSION-The results suggest that overweight and obese women are more likely to introduce complementary foods early and that psychological factors during pregnancy do not influence this relationship. Future studies need to explore why overweight/obese women are less likely to meet the AAP recommendations for the introduction of complementary food.

\section{Keywords}

Overweight; obesity; pregravid body mass index; complementary food introduction

\section{INTRODUCTION}

The American Academy of Pediatrics (AAP) recommends that infants be introduced to complementary foods after four months of age, preferably around six months, when they are physiologically ready for foods other than breast milk or human milk substitutes (1). Yet, $40 \%$ of infants in the United States (U.S.) are introduced to infant cereal by the time they are four months old (2). There is growing evidence that pregravid weight status may be a risk factor for adherence to infant feeding guidelines. Recent studies suggest that women who begin pregnancy overweight or obese are less likely to breastfeed and more likely to breastfeed for a shorter duration than women who begin pregnancy at a normal body mass index (BMI) (3-7). Research on the effects of pregravid BMI status on complementary food introduction is limited to two studies which are suggestive of a negative association between pregravid BMI and early food introduction $(8,9)$. These studies, however, did not comprehensively adjust for factors, such as sociodemographics, which might be the actual underlying drivers of the relationship between pregravid BMI and age of complementary food introduction. Further, it is unclear whether the findings from the studies, conducted in Europe, are generalizable to the U.S. population.

Improving adherence to infant feeding guidelines requires insight into why some women do not follow these recommendations. One pathway from obesity to infant feeding may be psychological but research of a possible mediatory pathway is lacking. There is evidence that obesity before pregnancy increases risk of poor mental health status during pregnancy $(10,11)$ which can persist well into the postpartum period (12) and negatively influence parenting practices $(13,14)$. Mothers with higher levels of psychological factors may be less likely to engage in positive parenting behaviors such as playing and talking with the child, establishing routines, and following infant feeding guidelines $(13,14)$. Several studies suggest a link between high levels of psychological factors during pregnancy and increased risk of early breastfeeding cessation (15-18). One study has examined the association between psychological factors during pregnancy and complementary foods; Hampson and colleagues found that negative affect, a combination of depression and anxiety, was associated with early introduction of solid foods despite controlling for pregravid BMI (9).

The aims of this analysis were to 1) determine the association between pregravid BMI status and timing of complementary food introduction and, 2) explore partial mediation by psychological factors. Compared with women of normal BMI, entering pregnancy overweight or obese was predicted to be associated with earlier introduction of 
complementary foods. In addition, the presence of depressive symptoms, stress and anxiety during pregnancy was hypothesized to partially mediate the effect of BMI status on early introduction.

\section{METHODS}

The Pregnancy, Infection and Nutrition Study (PIN) was a prospective study from 2001 to 2005 focusing on risk factors for preterm birth (19). Women between 15 to 20 gestational weeks $(n=2006)$ were recruited during their second prenatal clinic visit at the University of North Carolina (UNC) hospitals and followed through pregnancy. A postpartum component (PINPost) examined diet, physical activity and infant feeding (19). Participants in the PIN study were eligible for PINPost if they delivered a live-born infant between October 2002 and December 2005 and resided within a 2-hour radius from UNC in order to facilitate home visits $(n=1169)$. Those who agreed to participate in PINPost were interviewed in their homes by trained staff at three $(n=688)$ and 12 months postpartum $(n=550)$. This study analyzed data from 550 women who provided written consent and participated in both the three and 12 month postpartum visits. Further information on the PIN study and attrition from the PIN to the PINPost study is available in a previously published paper (19). Protocols for the prenatal and postpartum studies as well as this analysis were approved by the University of North Carolina Medical Institutional Review Board.

\section{Outcome}

The outcome variable, age of complementary food introduction, was created based on a composite of questions asked at the three and 12 month interviews. For each month leading up to the three month interview, women were asked, "How many times a day (24 hours) was your baby fed these foods during each of these months?" Women responded for the following foods: breast milk, infant formula, cow's milk, soy milk, cereals, tea, juice, fruits or vegetables, and meats. At the 12 month interview, women were asked, "At any time since the three month interview, have you fed your baby [type of food]?" for each month between the three and 12 month interviews. Women reported on the following foods: breast milk, infant formula, cow's milk, soy milk, cheese/yogurt, ice cream, infant cereals, cereals, breads, crackers, cookies (includes teething biscuits), tea, $100 \%$ fruit or vegetable juice, fruit drinks/Kool-Aid, fruits, vegetables, meats, fish, eggs, French fries, and soda. Age of complementary food introduction was categorized as follows: introduction of complementary foods less than four months of age, four to six, and six months or later (referent). Cutpoints for the categories reflect the unclear guidelines set by the AAP (1). Although the guidelines recommend that women should exclusively breastfeed until six months of age, they also state that complementary foods may be introduced as early as four months based on the "unique needs or feeding behaviors of the individual infants" (1).

\section{Exposure}

The main exposure was pregravid BMI, calculated from self-reported weight and height measured during screening. After checking weight for implausible values, three participants were excluded from analysis. There remained 547 women with recorded pregravid BMI and age of complementary food introduction. Pregravid BMI was categorized according to criteria of the World Health Organization for underweight $\left(<18.5 \mathrm{~kg} / \mathrm{m}^{2}\right)$, normal weight $\left(18.5 \mathrm{~kg} / \mathrm{m}^{2}\right.$ to $\left.24.9 \mathrm{~kg} / \mathrm{m}^{2}\right)$, and overweight/obese $\left(\geq 25.0 \mathrm{~kg} / \mathrm{m}^{2}\right)(20)$.

\section{Psychological variables}

The Center for Epidemiologic Studies-Depression scale (CES-D), completed by 490 participants ( $89 \%$ of 550), was a mail-in questionnaire provided at the second prenatal visit between 24 and 29 weeks' gestation (21). A composite score was calculated and scores of 
17 or higher indicated the presence of a significant level of depressive symptoms in the previous week. The Perceived Stress Scale (PSS), administered during a phone interview conducted between 27 and 30 weeks gestation, measured the extent to which participants (95.8\% of 550) found situations in their lives to be stressful in the last month (22). Scores were summed across items and categorized as follows: 0 to $<11$ (low perceived stress, referent); 11 to $<17$ (moderate), and $\geq 17$ (high). Participants (88.5\% of 550) completed the State-Trait Anxiety Inventory (STAI) (23) as part of a mail-in questionnaire provided at the second prenatal visit between 24 and 29 weeks gestation. This analysis used data from the state-anxiety section of the scale which assessed how the participant felt at the time of the interview. The variable was categorized into three levels: 0 to $<29$ (low anxiety; referent), 29 to $<39$ (moderate anxiety), and $\geq 39$ (high anxiety). The CES-D, PSS, and the STAI are validated instruments that have been used extensively in diverse populations, including among pregnant women $(21,24-26)$.

\section{Covariates}

Data on covariates were collected through interviews, self-reported questionnaires, and medical chart abstraction. Although the PIN and PINPost studies collected data on a wide variety of variables, only the following were examined as potential confounders. During pregnancy, participants reported sociodemographic factors such as race (categorized as nonWhite versus White), age, parity (primiparous versus nulliparous), family income, household size, education ( $<12$ years completed versus $\geq 12$ years), and marital status (not married versus married). Information on family income and household size was used to create a variable representing percent of the 2001 poverty index according to the U.S. Bureau of the Census (27). It was categorized as $<185 \%$ of the 2001 poverty line, $185 \%$ to $350 \%$, and $\geq 350 \%$.

\section{Statistical Analyses}

The regression analyses were restricted to participants for whom there was complete information on pregravid BMI and age of complementary food introduction $(n=547)$; the mediation analyses were further limited to those who completed all three psychological assessments $(n=470)$. The association between pregravid BMI and age of complementary food introduction was originally modeled using ordinal logistic regression but because the proportional odds assumption was violated for all explanatory factors, including the main exposure, the multinomial logit model was utilized instead. Multinomial regression was used to estimate relative risk ratios (RRR) of the association between pregravid BMI and complementary food introduction. For mediation analysis, binomial regression models were used where the outcome was dichotomous and multinomial logit models were utilized where the outcome was polytomous. Potential confounders were chosen based on a directed acyclic graph created from a review of the literature (28) and on the strength of their relationship with exposure and outcome. The sample size was not large enough to test for effect measure modification. The adjusted model was built using backward elimination with confounders kept in the model if they changed the beta coefficients of the exposure categories by more than $10 \%$.

Mediation analysis was comprised of a series of regression analyses (29). To be considered a mediator, the exposure must be associated with the outcome; the mediator must be predicted by the exposure; the outcome must be predicted by the mediator while adjusting for the exposure; and the effect estimate of the exposure must be reduced when adjusting for the mediator. Depressive symptoms, perceived stress and anxiety were examined in separate mediation analyses. All statistical analyses were conducted using Stata software (version 9.2, 2007, StataCorp, College Station, TX). 


\section{RESULTS}

The majority of the 550 participants (75.3\%) followed from pregnancy to 12 months postpartum introduced complementary foods when the infant was four to six months old; $19.6 \%$ introduced before four months of age and $5.1 \%$ after six months. At four and six months postpartum, $65.5 \%$ and $56.7 \%$ of women were breastfeeding, respectively. Women who introduced complementary foods before four months of age were more likely to be nonWhite, overweight/obese before pregnancy, multiparous, unmarried, less educated, and of lower income (Table 1). They were also less likely to have initiated breastfeeding or to be breastfeeding at the three month interview.

Of 550 infants, $19.6 \%$ had been introduced to complementary foods before four months of age and $67.8 \%$ before six months. Infant cereal was the most common food given to infants; $17.8 \%$ were fed infant cereal before four months of age and $61.8 \%$ by six months. Few infants were introduced to fruits/vegetables (5.5\%) before they were four months old but by six months, $75.5 \%$ had been fed fruits/vegetables.

Twenty-one percent of 490 women who completed the CES-D had high levels of depressive symptoms, 25.8\% (of 527) had high levels of perceived stress and 22\% (of 487) had high levels of state anxiety. High levels of depressive symptoms, stress, and anxiety during pregnancy were significantly more likely among women who began pregnancy overweight or obese (compared to normal weight) and those who introduced complementary foods before four months of age (compared with six months or later).

Results from an unadjusted model of pregravid BMI and age of complementary food introduction revealed that women who were overweight or obese before pregnancy were four times as likely $[R R R=4.00$ (95\% CI: $2.37,6.74)]$ to introduce complementary foods before the infant was four months old compared with normal weight women (Table 2). After adjusting for race, education, and poverty status, the risk estimate attenuated but remained significant $[R R R=2.22(1.23,4.01)]$.

\section{Mediation}

A series of crude models revealed all three psychological factors to be weak mediators (supplemental tables are available from the authors). The effect of pregravid BMI on age of introduction was reduced $6.8 \%$ by depressive symptoms, $6.2 \%$ by anxiety, and $3.3 \%$ by stress. After including race, education and poverty level in the mediation models, the psychological factors were no longer predicted by pregravid BMI status nor associated with the outcome. Thus, they could not be considered mediators.

\section{DISCUSSION}

This analysis identified maternal overweight/obesity before pregnancy as a risk factor for the early introduction of complementary foods. The results suggest that women who enter pregnancy overweight or obese are more likely to introduce complementary foods to their infant before the recommended age of four months. Research on the associations between maternal obesity and infant feeding has focused more on outcomes related to breastfeeding behavior rather than the introduction of complementary foods (4-6,30,31). Two previous studies examining the association between pregravid BMI and complementary food introduction were conducted in populations not representative of the high obesity prevalence or racial/demographics of the U.S. population $(8,9)$. Despite differences in population and methodology, however, the results presented in this paper confirm findings from these studies of a positive association between pregravid overweight/obesity and early introduction of complementary foods. Additionally, the findings from this paper build upon 
two prior analyses conducted within the PIN study population which showed that overweight/obesity before pregnancy increased risk of not breastfeeding (32) and of shorter breastfeeding duration (7). When considered together, results from the three papers support a strong negative association between pregravid overweight/obesity and lower adherence to infant feeding guidelines.

In addition, this paper adds to the current literature through its exploration of mediation by psychological factors. There may be several reasons for why the psychological factors were not found to mediate the association between pregravid BMI and complementary food introduction. First, the study experienced attrition. Women who did not participate in PINPost had significantly higher levels of depressive symptoms and anxiety $(p<0.05)$ which may have reduced the ability to find an association with complementary food introduction. Second, it may be that clinically assessed depression and anxiety would be more strongly associated with the exposure and outcome, producing a mediatory pathway that could exist despite adjustment for confounding. Third, because this is an observational study, causal inferences cannot be made and bidirectionality must be considered. And, finally, it may be that psychological factors during pregnancy do not strongly influence women's decisions on infant feeding. This is supported by two previously published papers which also found no mediation by psychological factors in the association between pregravid BMI and breastfeeding initiation/duration $(7,32)$.

An additional limitation is the calculation of pregravid BMI using weight that was selfreported during pregnancy. Because women of childbearing age tend to underestimate their weight (33), some BMI values may be artificially low. However, all pregravid BMI values were checked for implausibility and any potential misclassification was further minimized by categorizing the BMI variable. These findings are also limited in their generalizeability to the overall U.S. population because the study consisted primarily of women that were Caucasian, of higher socioeconomic status, and received prenatal care. The study population was representative of the racial demographics of North Carolina (34) but the small sample size restricted the ability to examine modification by race. This is an important point for future research given that nonwhites, specifically African Americans, have a much higher rate of obesity and depression and have been found to introduce foods in early postpartum (35-37).

\section{CONCLUSIONS}

This analysis revealed a strong, inverse association between pregravid BMI and age of complementary food introduction and showed that this association was not explained by psychological factors. The results highlight the need for a tailored intervention targeting overweight and obese women to delay the introduction of complementary foods until at least four months of age. Women who are overweight or obese at the start of pregnancy may require more counseling on infant feeding recommendations and greater guidance on adhering to them than normal weight women. To provide counseling that is effective in both raising awareness of and adherence to infant feeding guidelines, a better understanding of why overweight and obese women are less likely to follow these recommendations is necessary. Future studies need to confirm the associations found in this study in a larger and more diverse sample population and explore reasons that may provide a more in-depth understanding of the pregravid BMI-infant feeding relationship.

\section{REFERENCES}

1. Gartner LM, Morton J, Lawrence RA, et al. Breastfeeding and the use of human milk. Pediatrics. 2005; 115(2):496-506. [PubMed: 15687461] 
2. Grummer-Strawn LM, Scanlon KS, Fein SB. Infant feeding and feeding transitions during the first year of life. Pediatrics. 2008; 122(Suppl 2):S36-S42. [PubMed: 18829829]

3. Rasmussen KM, Hilson JA, Kjolhede CL. Obesity as a risk factor for failure to initiate and sustain lactation. Adv Exp Med Biol. 2002; 503:217-222. [PubMed: 12026023]

4. Hilson JA, Rasmussen KM, Kjolhede CL. High prepregnant body mass index is associated with poor lactation outcomes among white, rural women independent of psychosocial and demographic correlates. J Hum Lact. 2004; 20(1):18-29. [PubMed: 14974697]

5. Baker JL, Michaelsen KF, Sørensen TIa, Rasmussen KM. High prepregnant body mass index is associated with early termination of full and any breastfeeding in Danish women. Am J Clin Nutr. 2007; 86(2):404-411. [PubMed: 17684212]

6. Li R, Jewell S, Grummer-Strawn L. Maternal obesity and breast-feeding practices. Am J Clin Nutr. 2003; 77(4):931-936. [PubMed: 12663294]

7. Mehta UJ, Siega-Riz AM, Herring AH, Adair LS, Bentley ME. Pregravid body mass index, psychological factors during pregnancy and breastfeeding duration: Is there a link? Matern Child Nutr. 2011

8. Baker JL, Michaelsen KF, Rasmussen KM, Sørensen T. Maternal prepregnant body mass index, duration of breastfeeding, and timing of complementary food introduction are associated with infant weight gain. Am J Clin Nutr. 2004; 80(6):1579-1588. [PubMed: 15585772]

9. Hampson SE, Tonstad S, Irgens LM, Meltzer HM, Vollrath ME. Mothers' negative affectivity during pregnancy and food choices for their infants. Int J Obes (Lond). 2010; 34(2):327-331. [PubMed: 19918247]

10. Bodnar LM, Wisner KL, Moses-Kolko E, Sit DK, Hanusa BH. Prepregnancy body mass index, gestational weight gain, and the likelihood of major depressive disorder during pregnancy. J Clin Psychiatry. 2009; 70:1290-1296. [PubMed: 19607761]

11. Laraia BA, Siega-Riz AM, Dole N, London E. Pregravid weight is associated with prior dietary restraint and psychosocial factors during pregnancy. Obesity (Silver Spring). 2009; 17(3):550 558. [PubMed: 19131943]

12. American Psychiatric Association. Diagnostic and statistical manual of mental disorders. 4th ed.. Washington, DC: American Psychiatric Publishing; 2000. p. 943

13. McLearn KT, Minkovitz CS, Strobino DM, Marks E, Hou W. Maternal depressive symptoms at 2 to 4 months post partum and early parenting practices. Arch Pediatr Adolesc Med. 2006; 160(3): 279-284. [PubMed: 16520447]

14. Hurley KM, Black MM, Papas MA, Caufield LE. Maternal symptoms of stress, depression, and anxiety are related to nonresponsive feeding styles in a statewide sample of WIC participants. J Nutr. 2008; 138(4):799-805. [PubMed: 18356338]

15. Hatton DC, Harrison-Hohner J, Coste S, Dorato V, Curet LB, McCarron DA. Symptoms of postpartum depression and breastfeeding. J Hum Lact. 2005; 21(4):444-449. quiz 450-4. [PubMed: 16280561]

16. Dennis CL, McQueen K. Does maternal postpartum depressive symptomatology influence infant feeding outcomes? Acta Paediatr. 2007; 96(4):590-594. [PubMed: 17391475]

17. Dennis CL, McQueen K. The relationship between infant-feeding outcomes and postpartum depression: A qualitative systematic review. Pediatrics. 2009; 123(4):e736-e751. [PubMed: 19336362]

18. Dunn S, Davies B, McCleary L, Edwards N, Gaboury I. The relationship between vulnerability factors and breastfeeding outcome. JOGNN. 2006; 35(1):87-97. [PubMed: 16466356]

19. Siega-Riz AM, Herring A, Carrier K, Evenson KR, Dole N, Deierlein A. Sociodemographic, perinatal, behavioral, and psychosocial predictors of weight retention at 3 and 12 months postpartum. Obesity. 2009; 18:1996-2003. [PubMed: 20035283]

20. World Health Organization. Obesity: Preventing and managing the global epidemic. report of a WHO consultation. World Health Organ Tech Rep Ser. 2000; 894:i-xii. 1-253.

21. Radloff LS. The CES-D scale: A self-report depression scale for research in the general population. Appl Psychol Measure. 1977; (1):385-401. 
22. Cohen, S.; Williamson, GM. Perceived stress in a probability sample of the United States. In: Spacapam, S.; Oscamp, S., editors. The social psychology of health. Newbury Park, CA: Sage; 1988.

23. Spielberger, CD. Manual for the state-trait anxiety inventory. Palo Alto, CA: Consulting Psychologists Press; 1983.

24. Maloni JA, Park S, Anthony MK, Musil CM. Measurement of antepartum depressive symptoms during high-risk pregnancy. Res Nurs Health. 2005; 28(1):16-26. [PubMed: 15625706]

25. Cohen S, Kamarck T, Mermelstein R. A global measure of perceived stress. J Health Soc Behav. 1983; 24(4):385-396. [PubMed: 6668417]

26. Gunning MD, Denison FC, Stockley CJ, Ho SP, Sandhu HK, Reynolds RM. Assessing maternal anxiety in pregnancy with the State-Trait Anxiety Inventory (STAI): Issues of validity, location and participation. Journal of Reproductive and Infant Psychology. 2010; 28(3):266-273.

27. Proctor, BD.; Dalaker, J. U.S. Census Bureau. Washington, DC: U.S. Government Printing Office; 2002. Current population reports. Poverty in the United States: 2001. Series P60-219:p.5

28. Greenland S, Pearl J, Robins JM. Causal diagrams for epidemiologic research. Epidemiology. 1999; 10(1):37-48. [PubMed: 9888278]

29. Baron RM, Kenny DA. The moderator-mediator variable distinction in social psychological research: Conceptual, strategic, and statistical considerations. J Pers Soc Psychol. 1986; 51(6): 1173-1182. [PubMed: 3806354]

30. Donath SM, Amir LH. ALSPAC Study Team. Relationship between prenatal infant feeding intention and initiation and duration of breastfeeding: A cohort study. Acta Paediatr. 2003; 92(3): 352-356. [PubMed: 12725552]

31. Oddy WH, Li J, Landsborough L, Kendall GE, Henderson S, Downie J. The association of maternal overweight and obesity with breastfeeding duration. J Pediatr. 2006; 149(2):185-191. [PubMed: 16887431]

32. Mehta UJ, Siega-Riz AM, Herring AH, Adair LS, Bentley ME. Maternal obesity, psychological factors, and breastfeeding initiation. Breastfeed Med. 2011; 6:369-376. [PubMed: 21492019]

33. Brunner Huber LR. Validity of self-reported height and weight in women of reproductive age. Matern Child Health J. 2007; 11(2):137-144. [PubMed: 17066316]

34. U.S. Census Bureau. [Accessed July 2, 2010] State \& county quickfacts: North carolina. http://quickfacts.census.gov. Updated April 22, 2010

35. Centers for Disease Control and Prevention. [Accessed May 31, 2010] Breastfeeding report card, United States - 2009: Outcome indicators.

http://www.cdc.gov/breastfeeding/data/report_card2.htm. Updated 2009

36. Flegal KM, Carroll MD, Ogden CL, Curtin LR. Prevalence and trends in obesity among US adults, 1999-2008. JAMA. 2010; 303(3):235-241. [PubMed: 20071471]

37. Bronner YL, Gross SM, Caulfield L, et al. Early introduction of solid foods among urban AfricanAmerican participants in WIC. J Am Diet Assoc. 1999; 99(4):457-461. [PubMed: 10207399] 


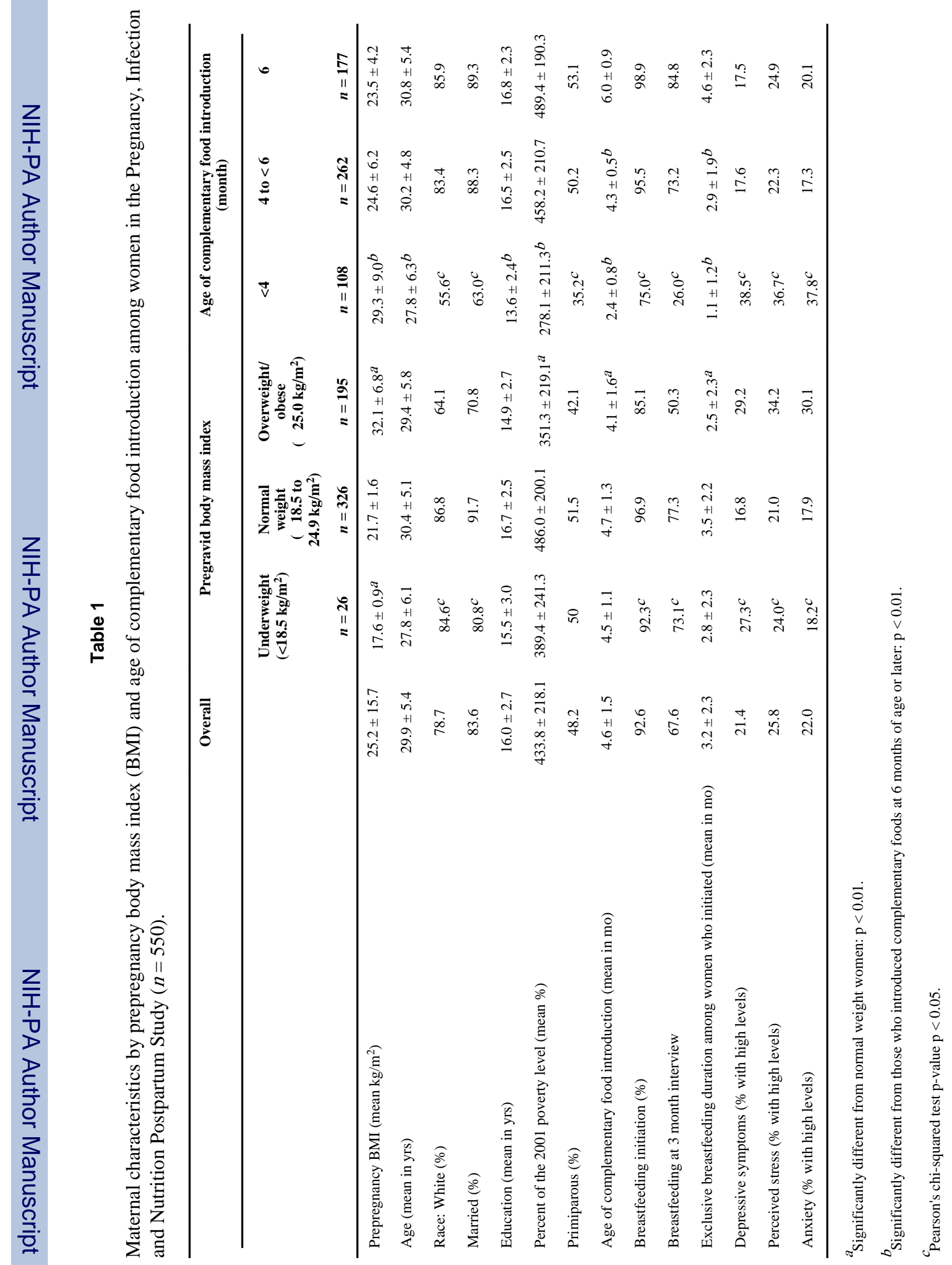




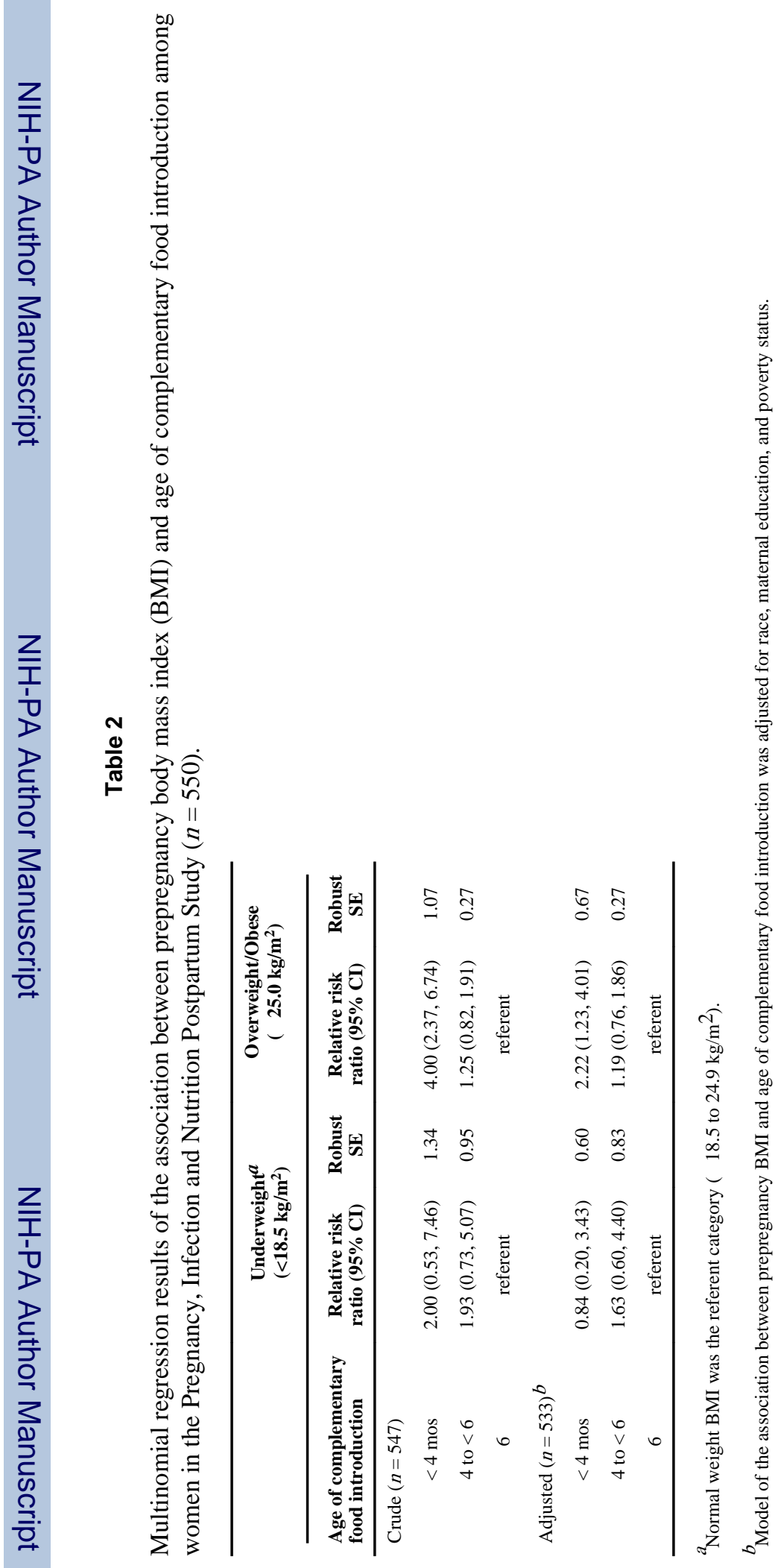

